\section{South-seeking magnetotactic bacteria in the Southern Hemisphere}

\author{
R. P. Blakemore*, R. B. Frankel ${ }^{\dagger}$ \\ \& Ad. J. Kalmijn $\ddagger$
}

* Department of Microbiology, University of New Hampshire, Durham, New Hampshire 03824

$\checkmark$ Francis Bitter National Magnet Laboratory, Massachusetts Institute of Technology, Cambridge, Massachusetts ()2139

$\doteqdot$ Department of Biology, Woods Hole Oceanographic Institution, Woods Hole, Massachusetts 02543

Several species of aquatic bacteria which orient in the Earth's magnetic field and swim along magnetic field lines in a preferred direction (magnetotaxis) have been observed in marine and freshwater sediments of the Northern Hemisphere ${ }^{1,2}$. Their orientation is due to one or more intracytoplasmic chains of single-domain magnetite particles ${ }^{3}$. These linearly arranged particles impart a net magnetic dipole moment to the bacterium, parallel to the axis of motility. Northern Hemisphere magnetotactic bacteria with unidirectional motility swim consistently in the direction of the magnetic field, that is, to the geomagnetic North $^{1,2.4}$. This implies that their magnetic dipole is systematically orientated with the North-seeking pole forward. The magnetic polarity can be reversed by single, magnetic pulses of high field strength (1-2 $\mu \mathrm{s}, 300-600 \mathrm{G}$ ), and these bacteria then swim along magnetic field lines to the South ${ }^{5}$. Due to the inclination of the Earth's magnetic field, magnetotactic bacteria which swim to the North in the Northern Hemisphere are directed downward at an angle increasing with latitude. It has been suggested that this downward-directed motion confers a biological advantage by guiding the bacteria, when dislodged, back to the sediments ${ }^{\prime}$. On the basis of this hypothesis, magnetotactic bacteria of the Southern Hemisphere would be expected to swim to the South to reach the bottom. We report here several morphological types of magnetotactic bacteria present in sediments of the Southern Hemisphere. These bacteria indeed swim consistently to the South, hence downward along the Earth's inclined magnetic field lines, as hypothesized. As revealed by electron microscopy, they contain internal chains of electron-opaque particles similar to those observed in magnetotactic bacteria from the Northern Hemisphere. Like their Northern Hemisphere counterparts, their magnetic polarity can be permanently reversed and they cannot be demagnetized. We also report on Northern Hemisphere magnetotactic bacteria incubated in Southern Hemisphere magnetic conditions, confirming the biological relevance of downward directed motility.

Bacteria were collected from marine and freshwater sediments in the vicinities of Christchurch. New Zealand, and Hobart, Tasmania, Australia. These locales offered ecologically diverse halitats with geomagnetic characteristics (inclination $68-73^{\circ} \mathrm{S}$ at a total magnetic intensity of $0.59-0.63 \mathrm{G}$ ) approximately the mirror image of Northern Hemisphere environments where magnetotactic hacteria are found. Magnetotactic responses were recorded by optically monitoring hacterial motion in uniform magnetic fields of $0.2-2 \mathrm{G}$, provided by a pair of Helmholtz coils mounted to either side of a Zeiss OPMI 1 operation microscope (magnification $\times 83.2$; working distance, $10 \mathrm{~cm})$. The Helmholtz-coil axis was aligned with the horizontal component of the Earth's magnetic field; the polarity of the imposed field was selected by a toggle switch. The coils and the dark-field illuminator were hattery operated. In the coil system, both freshwater and marine bacteria migrated along magnetic field lines in the direction opposite to that indicated by a magnetic compass needle. On reversal of the field, the organisms executed $U$-turns and swam opposite to the initial direction. The rates of migration were comparable to those observed in
Northern Hemisphere bacteria. Thus, the magnetotactic responses of Southern Hemisphere bacteria are similar to those of the Northern Hemisphere species, except for their magnetically opposite direction of migration (South-seeking magnetotactic bacteria have also been observed in Victoria, Australia ${ }^{6}$ ).

Transmission electron microscopy of bacteria magnetotactically separated from sediments was carried out at the University of Canterbury, Christchurch, New Zealand, using a Hitachi HS-7S electron microscope operating at $50 \mathrm{kV}$. Samples were placed on parlodion-coated, carbon-reinforced, 300 -mesh copper grids, and were lightly stained with uranyl acetate. Magnetic cells of various morphological types were observed to contain one or two chains of intracytoplasmic electron-opaque particles which vary in size and shape with cell type (Fig. 1). Thus, Southern Hemisphere magnetotactic bacteria also possess

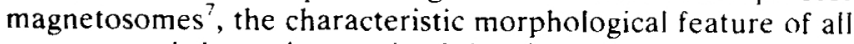
magnetotactic bacteria examined thus far.

To establish the ferromagnetic nature of their orientation mechanism, bacteria were subjected to alternating $50-\mathrm{Hz}$ magnetic fields over $1,000 \mathrm{G}$, produced by a small hand-held magnetic tape degausser (Tandy Corporation). Northern Hemisphere and Southern Hemisphere magnetotactic bacteria were examined in separate water drops on a microscope slide before and after exposure to the alternating magnetic field. On exposure, the degausser was slowly moved away from the water drops to obtain the field decay required for demagnetization. Whereas before exposure all the cells in these samples swam exclusively either to the North or to the South, after exposure

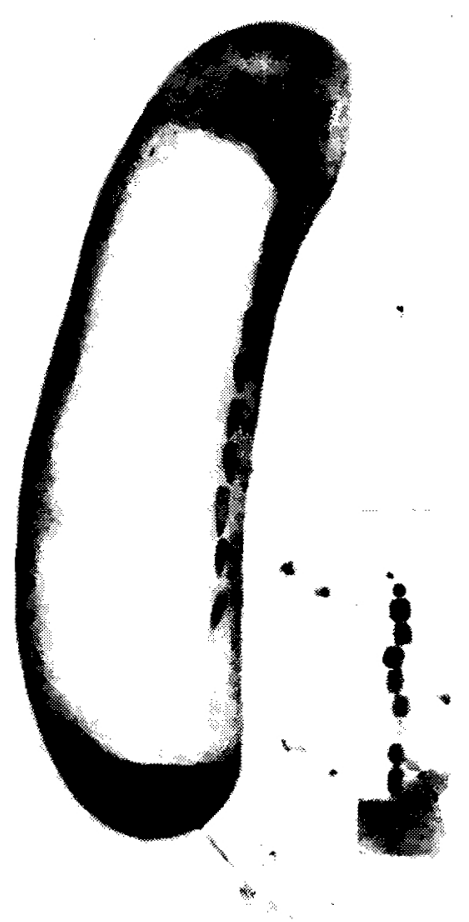

Fig. I Electron micrograph of a magnetotactic bacterium separated magnetically from freshwater sediments of New Zealand and stained with uranyl acetate. Inset show one of two chains of particles found in a freshwater maynetotactic coccus of $\mathrm{New}$ Zealand. Both photographs are presented at the same magnification (scale bar $=1 \mu \mathrm{m}$ ). Whereas the particles shown in the inset are similar to those found in Vorthern Hemisphere magnetotactic hacteria, those in the rod-shaped species have at novel shape. 
each sample contained approximately equal numbers of Northand South-seeking cells. Magnetic reversals were also effected by approaching individual hacteria at the water-air interface with a sharp, magnetized needle. These observations indicate that bacteria from both hemispheres physically function as single-domain magnetic dipoles, in agreement with previous remagnetization studies using strong magnetic pulses of short duration ${ }^{5}$

In experiments carried out in Massachusetts, sediment samples containing North-seeking bacteria from New England were placed in an experimental chamber in which the vertical component of the local Earth's magnetic field was inverted, reproducing conditions in the Southern Hemisphere. Control samples were placed in a similar chamber with a field of normal inclination. The polarities of cells from the samples were determined periodically for several weeks, that is, over many generations. Whereas all samples contained almost exclusively North-seeking cells at the beginning of the experiment, with time the number of North-seeking cells in the experimental chamber decreased dramatically, and South-seeking bacteria gradually predominated. In the control samples no such changes occurred. Thus, downward-inclined fields select for Northseeking bacteria whereas upward-inclined fields select for South-seeking cells. This demonstrates that the vertical component of the Earth's magnetic field is the relevant parameter determining the predominant cell polarity.

In conclusion, the prevalence of South-seeking magnetotactic bacteria in Southern Hemisphere sediments and in samples held in similar magnetic conditions in the Northern Hemisphere verifies the hypothesis that downward-directed motion is advantageous for and upward-directed motion detrimental to the survival of these magnetotactic bacteria with unidirectional motility. Magnetotaxis is a reliable means of keeping these microorganisms in or near the bottom sediments. As particles in the micrometre size range with densities close to 1.0 tend to remain suspended in water, gravity is virtually inconsequential in determining the vertical distribution of the bacteria.

The Earth s magnetic field provides a global orientation cue to which various organisms are known to respond. Only recently have detection mechanisms based on electromagnetic induction $^{x}$ and ferromagnetic alignment ${ }^{5.9}$ been explained. In the bacteria, nature has shown the biological feasibility of synthesizing a highly organized ferromagnetic structure equivalent to a magnetic compass needle.

We thank Nancy Blakemore for her participation in the expedition 10 New Zealand and Tasmania, Australia. Drs $T$ Cole, L. Greenfield and W. D. Parkinson for hospitality and co-operation, M. Ingerfeld for assistance with electron microscopy, and H. Stram, D. Lobel, J. Pero, V. Kalmijn and T Dourdeville for valuable contributions to this work. This work was supported by a research grant from the National Geographic Society, and the Office of Naval Research. The Francis Bitter National Magnet Laboratory is supported by the NSF and R.P.B. is supported, in part, by NSF grant PCM77-12175.

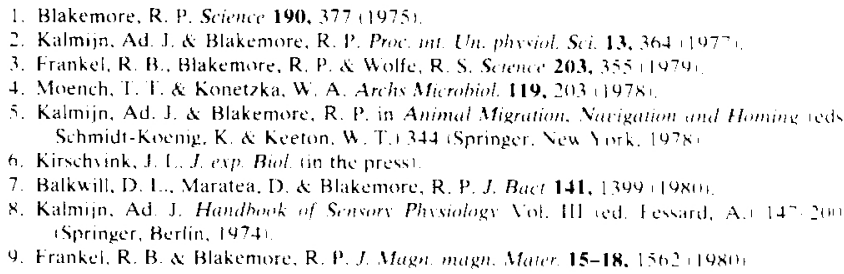

